\title{
Ações docentes nos Ambientes Virtuais de Aprendizagem proporcionadas pelas ferramentas de Learning Analytics
}

\author{
Maurício Vieira Dias Júnior ${ }^{1}$ (1) @ \\ Luís Paulo Leopoldo Mercado' (1) (a) \\ ${ }^{1}$ Universidade Federal de Alagoas (UFAL), Brasil
}

Resumo. Percebe-se que há uma relevante adoção dos ambientes virtuais de aprendizagem (AVA) na educação superior, em especial na modalidade a distância. Estes ambientes, com o apoio do Big Data, podem proporcionar ações para a prática avaliativa docente a partir de ferramentas que contemplem os objetivos da learning analytics (LA), segundo modelos e ciclos de vida já definidos na literatura. Neste estudo foi desenvolvida a consolidação de dez possíveis ações docentes a partir destas ferramentas, modelos e ciclos de vida, tendo como objetivo a confrontação destas ações com docentes envolvidos oriundos de instituições de ensino superior (IES) que atuam na educação a distância (EaD), evidenciando-as através de um experimento onde utilizaram seis destas ferramentas de perfil docente em suas respectivas disciplinas no AVA Moodle. Para coleta, foram utilizados dois questionários semi-estruturados (pré e pós-experimento). Ficou caracterizado como resultados deste estudo que as ferramentas que tiveram maior destaque para ações de LA foram: Completion Progress (18,5\%), Course Dedication (18,1\%) e Level Up! (17,8\%), já as ações de LA com maiores graus de destaque foram: "monitoramento" (17,4\%), seguido por "avaliação/ feedback" (13,0\%) e "intervenção" (12,0\%), havendo assim, compatibilidade com o propósito de atingir a prática avaliativa dentro dos AVA a partir destas ferramentas.

Palavras-chave: learning analytics, ambientes virtuais de aprendizagem, educação a distância, educação superior, big data.

Acciones docentes en los entornos virtuales de aprendizaje proporcionados por las herramientas de Learning Analytics

Resumen. Empieza a percibirse una relevante adopción de los entornos virtuales de aprendizaje (EVA) en la educación superior, en especial en la modalidad a distancia. Estos ambientes, con el apoyo del big data, pueden proporcionar acciones para la práctica de evaluación del docente a partir de herramientas contempladas en los objetivos del Learning Analytics (LA), según modelos y ciclos de vida definidos en la literatura. En este estudio se desarrolló la consolidación de diez posibles acciones docentes a partir de estas herramientas, modelos y ciclos de vida. El objetivo fue la confrontación de estas acciones con un grupo de docentes involucrados, adscritos a instituciones de enseñanza superior (IES), que ejercen en la educación a distancia (EaD), evidenciándolas a través de un experimento, en el que se utilizaron seis de estas herramientas de perfil docente en sus respectivas disciplinas en el EVA Moodle. Para la recolección, se utilizaron dos cuestionarios semiestructurados (prey post-experimento). Se evidenció en los resultados de este estudio que las herramientas que tuvieron más relevancia para acciones de LA fueron: Completion Progress (18,5\%), Course Dedication (18,1\%) y Level Up! (17,8\%); las acciones de LA con mayor grado de destaque fueron: "monitoreo" (17,4\%), seguido por "evaluación / feedback" (13,0\%) e "intervención" (12,0\%), comprobándose así, que existe compatibilidad en el propósito de alcanzar la práctica de evaluación dentro de Ios EVA a partir de estas herramientas:

Palabras clave: learning analytics, entornos virtuales de aprendizaje, educación a distancia, educación universitaria, big data.

Teacher actions in Virtual Learning Environments provided by Learning Analytics tools Abstract. It is noticed that there is a relevant adoption of virtual learning environments (VLE) in higher education, especially in the distance modality. These environments, with the support of Big Data, can provide actions for teaching evaluation practice based on tools that meet the objectives of learning analytics (LA), according to models and life cycles already defined in the literature. The purpose of this study was to consolidate ten possible teaching actions based on these tools, models and life cycles, with the objective of confronting these actions with teachers involved from higher education 
institutions that work in distance education evidencing them through an experiment where they used six of these tools of teaching profile in their respective disciplines in VLE Moodle. For collection, two semi-structured questionnaires (pre and post-experiment) were used. It was characterized as results of this study that the tools that had the most highlight for LA actions were: Completion Progress (18.5\%), Course Dedication (18.1\%) and Level Up! (17.8\%), followed by "evaluation /feedback" (13.0\%) and "intervention" $(12.0 \%) \%)$, thus being compatible with the purpose of reaching the evaluative practice within VLE from these tools.

Keywords: learning analytics, virtual learning environments, distance learning, college education, big data.

\section{Introdução}

Surgidos na década de 90, concomitantemente com os primeiros navegadores da web, a partir de distintas mídias e linguagens de programação, os AVA representam uma grande contribuição para a história da educação (Silva, 2013), sendo instrumentos para proporcionar, por exemplo, atividades mais ricas de cunho colaborativo e interativo, baseado na web e considerado uma plataforma que se constrói e entrega módulo e cursos.

É percebido que há uma forte adoção desses AVA no mundo. Estes são utilizados extensivamente pelas IES (Ekuase-Anwansedo et al., 2018) a exemplo do Moodle que além de ser fácil de usar, de gerar conteúdo, engajar discente e sem necessidade de adquirir licenças, tem sido constantemente melhorado por especialistas técnicos (Dodun et al., 2015), sendo o mais adequado pelos quesitos de modularidade, popularidade, disponibilidade de plugins e por contar com uma forte comunidade de desenvolvedores (Medio et al., 2017).

Nota-se esta expansão no Brasil, não só pelo fator econômico, já que as IES podem adquiri-lo de forma gratuita, através da versão com software livre, mas também porque os conteúdos dos cursos a distância são melhores gerenciados e a maioria é customizada pela própria instituição segundo a sua demanda, sem a necessidade de ônus (ABED, 2017).

Porém o que se vê, conforme Silva et al. (2009) é que, no geral, os cursos oferecidos na modalidade de EaD que utilizam esses AVA, adotam abordagens tradicionais de avaliação, tendo maior peso nas provas finais presenciais, influenciadas pelo tecnicismo. Mesmo sendo autodenominados construtivistas, ficando assim com dificuldades em promover uma avaliação reflexiva, crítica e emancipatória. Em um processo de ensino passivo e repetitivo, essas atitudes são incompatíveis com a navegação nos AVA, sendo formal ou não. 
Também é notório que os métodos avaliativos em EaD sejam sempre revistos e aperfeiçoados, sendo uma preocupação constante em inúmeros estudos sobre esta temática (Polak, 2009). De acordo com Bitencourt et al. (2013) é preciso pensar formas mais concernentes de avaliação para este contexto online, pois se trata de uma forma peculiar de educação, a se comparar com as avaliações presenciais.

O Learning Analytics (LA), tendo big data como apoio fundamental, segundo Daniel (2015), representa novas oportunidades para a educação superior, podendo influenciar positivamente nas tomadas de decisões baseadas em evidências mais eficazes, possibilitando a realização de análises e avaliações em tempo real sobre as atividades educacionais.

O LA tem alcançado uma robusta posição entre outras tecnologias educacionais, sendo colocada como uma área com ganhos substanciais para potencializar o processo de ensino-aprendizagem (Khalil \& Ebner, 2015). Observa-se por parte dos atores da educação o interesse pela relevância que a LA tem para o apoio educacional, compartilhando análises para a ação e o efetivo aprendizado, sendo uma área interdisciplinar (Chatti et al., 2012).

A proposta deste estudo foi evidenciar as ações, apresentadas e definidas nos modelos (Chatti et al., 2012; Gasevic et al., 2017) e ciclos de vida (Clow, 2012; Khalil \& Ebner, 2015) da literatura, provenientes de ferramentas de LA do tipo plugin, que não são nativas, disponibilizadas, especificamente, para o AVA-Moodle, sendo estas utilizadas perante alguns docentes de IES que atuam na EaD.

\section{Learning Analytics}

A LA vem sendo discutida desde 2009, porém apenas em 2011 quando da ocasião da primeira ${ }^{1}$ International Conference on Learning Analytics and Knowledge (LAK), ocorrida no Canadá, foi definido o seu conceito sendo "medição, coleta, análise e relatórios de dados sobre aprendizes e seus contextos, para fins de compreensão e otimização da aprendizagem e dos ambientes em que ocorre" (Siemens, 2013, p. 1382), tendo como principal objetivo de melhorar a qualidade do aprendizado e do ensino.

George Siemens, um dos precursores da LA, e um dos organizadores do LAK, anunciou que LA não se trata apenas de análise de dados, mas incorpora também a ação de prever, através de ferramentas, a adaptação,

\footnotetext{
${ }^{1}$ https://tekri.athabascau.ca/analytics/
} 
a personalização e/ou intervenção, para antecipar as possíveis dificuldades posteriores a fim de melhorar o desempenho, durante o percurso do curso (Siemens, 2010).

Conforme a figura 1 , a LA através da sua principal conferência internacional LAK ${ }^{2}$ promovida pela Society for Learning Analytics Research (SoLAR), vem ampliando sua representação no quesito de submissões de artigos científicos ano a ano. Constata-se que no período de oito anos houve uma triplicação de artigos submetidos, de 38 em 2011 para 115 artigos submetidos no ano de 2018.

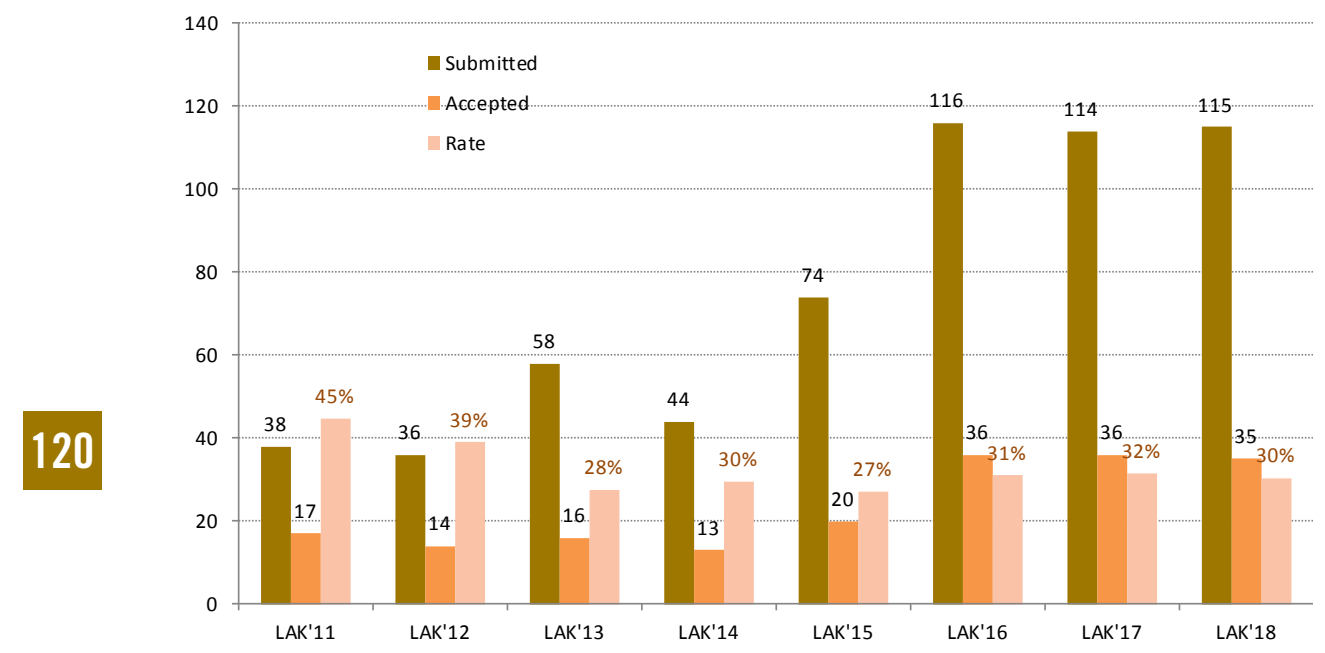

Figura 1. Crescimento das Conferências Internacionais LAK

Fonte: https://dl.acm.org/citation.cfm?id=3027385, acesso em 24 mar. 2019.

Desde o ano de 2014 a SoLAR também oferece uma publicação periódica oficial denominada Journal of Learning Analytics ${ }^{3}$. A LA foi mencionada pela primeira vez no relatório Horizon da New Media Consortium $(\mathrm{NMC})^{4}$ em 2012, que trata-se de uma comunidade internacional composta por centenas de relevantes universidades, faculdades, museus e centros de pesquisa, estimulando e promovendo a exploração e uso de novas mídias e tecnologias para aprendizagem e expressões criativas (Peña-Ayala, 2017).

\footnotetext{
${ }^{2}$ https://solaresearch.org/events/lak/

${ }^{3}$ https://learning-analytics.info

${ }^{4}$ https://www.nmc.org/nmc-horizon
} 
Na figura 2 é apresentado, inicialmente, um ciclo de vida fundamentado, que contempla holisticamente todos os aspectos de uma LA (Khalil \& Ebner, 2015).

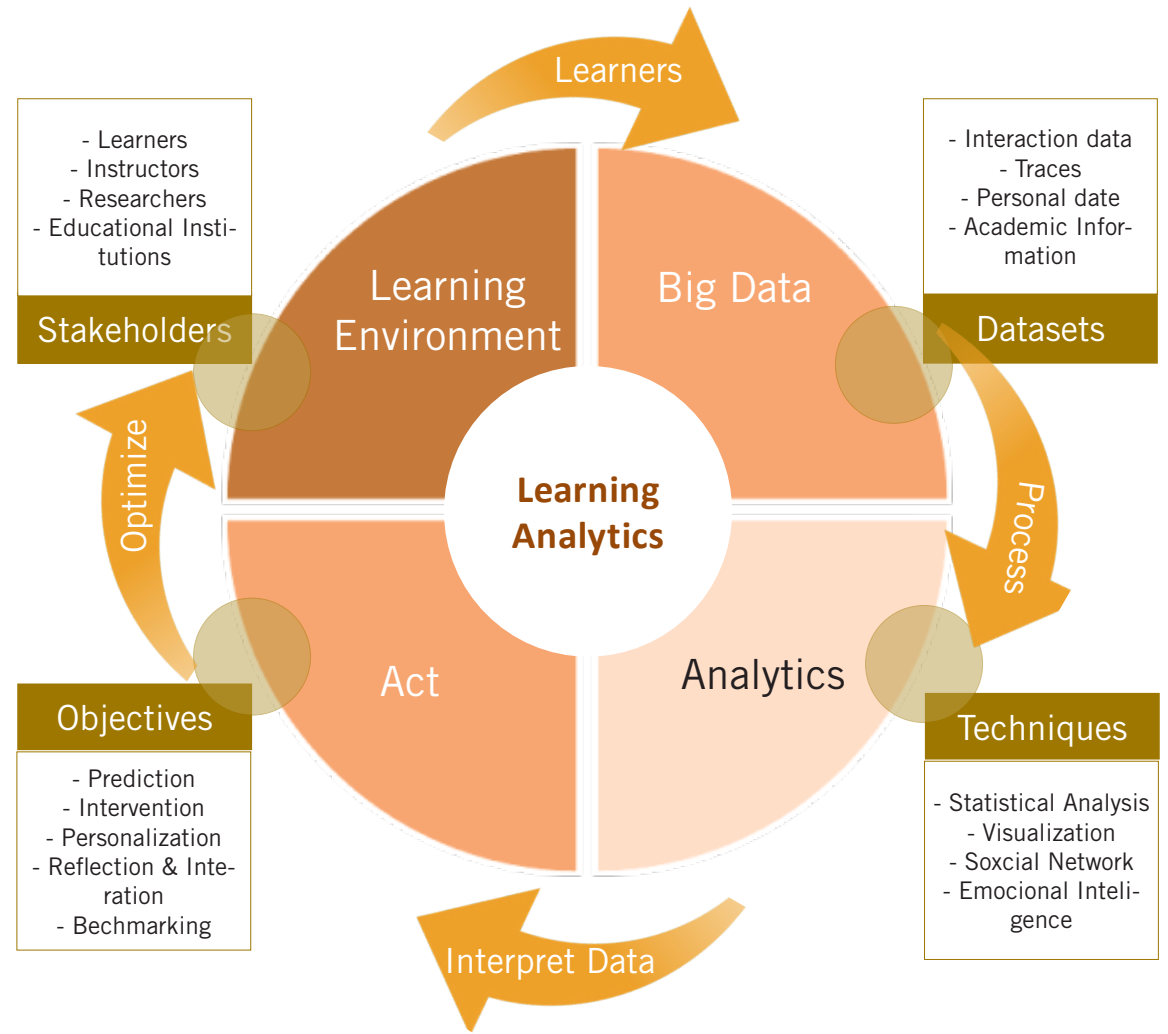

Figura 2. Proposta de ciclo de vida da LA.

Fonte: Khalil \& Ebner (2015)

No ciclo mostrado na figura 2 consolidado por Khalil e Ebner (2015), constam quatro tópicos principais que são: 1) os atores (stakeholders), que através de suas interações e produções nos AVA, geram os dados; 2) os conjuntos de dados que representam os Big Data contidos nos AVA, que armazenam os dados; 3 ) as diversas técnicas que fazem a análise dos dados; e 4) os objetivos que representam a ação que será aplicada para a otimização do desempenho da aprendizagem.

No domínio da educação, a LA torna-se um relevante instrumento nas mãos dos envolvidos nesta área, pois um vasto número de dados é gerado a partir da interação dos atores, dos conteúdos e da própria tecnologia nos AVA, sendo sistematicamente armazenados, os quais, consequentemente, poderão ser recuperados para descobrir conhecimento, possibilitando a melhoria na resolução de problemas educacionais. 


\section{Possíveis Ações com Learning Analytics nos Ambientes Virtuais de Aprendizagem}

Para além dos objetivos de organizar cursos e disciplinas, administrar conteúdos e monitorar os discentes, os AVA contém uma infraestrutura tecnológica com recursos suficientes para desempenhar uma abordagem pedagógica em um determinado curso (Machado et al., 2013).

Cientes da importância do real crescimento de LA, na qual os conjuntos de dados gerados a partir dos processos de aprendizagem dentro dos AVA são aproveitados para o aprimoramento educacional, foram construídos formalizações acerca de um modelo que descrevessem LA, tendo em vista as diversas conexões que LA tem com outras áreas.

A partir de uma investigação multidisciplinar, Chatti et al. (2012) desenharam um modelo de referência baseado em quatro dimensões: (1) dados e ambientes - quê tipo de dado será analisado?; (2) os atores - quem será o alvo da análise?; (3) os objetivos - porquê o sistema irá analisar os dados coletados? e; (4) os métodos - como serão analisados os dados coletados?, a fim de contemplar áreas que abrangem a LA, conforme mostrados na figura 3.

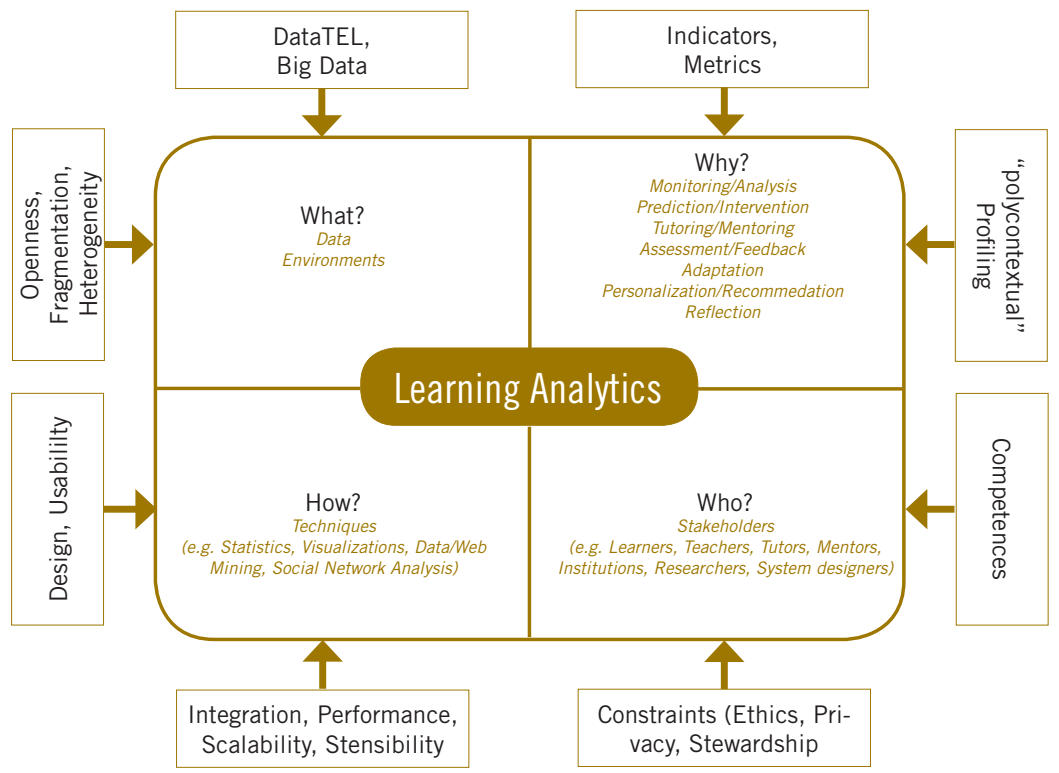

Figura 3. Modelo de referência para LA, baseado em quatro dimensões.

Fonte: Chatti et al. (2012, p. 7). 
Especificamente, quanto a terceira dimensão porquê?, que se refere aos objetivos do sistema em analisar os dados coletados, há diversos no qual pode-se citar (Chatti et al., 2012):

a) monitoramento e análise: que lida com acompanhamento das atividades desenvolvidas pelos discentes agregadas a relatórios e possibilitando alterações do design instrucional;

b) predição e intervenção: traçar um possível modelo para tentar prever a situação de desempenho discente no futuro, a fim de vislumbrar auxílios adicionais personalizados e eficazes para a melhoria do rendimento discente;

c) tutoria/mentoria: com a finalidade de controlar os discentes em algum processo de ensino ou durante todo o processo, supervisionando-os para o alcance das metas;

d) avaliação e feedback: para o apoio na auto-avaliação, obtendose também feedback inteligentes para os discentes e docentes;

e) adaptação: informar aos discentes os próximos passos, organizando os recursos e forma adaptativa;

f) personalização e recomendação: ajudando os discentes a decidirem como alcançar seus objetivos, sendo recomendado mudanças de comportamento e conteúdos, para uma aprendizagem auto-dirigida e, por fim, a;

g) reflexão: recurso importante que ajuda os atores a se autorefletirem sobre o seu andamento a partir de comparação de dados anteriores.

Retomando-se aqui e fazendo um elo com o ciclo de vida de LA definido por Khalil e Ebner (2015), quanto ao tópico 4, que se refere aos objetivos, correspondentes as ações que o docente pode ter provenientes das ferramentas de LA, tem-se também: a) predição, b) intervenção, c) recomendação, d) personalização, e) reflexão e iteração, f) benchmarking.

Conforme afirma Khalil e Ebner (2015), o maior valor de LA advém da otimização dos objetivos: oriundos da dimensão "Por quê?" de Chatti et al. (2012), na "intervenção" de Clow (2012) e no "learning design" de Gasevic et al. (2017) que proporcionam a geração de insights capazes de influenciar a prática avaliativa docente. 
Enquadrando-se na dimensão "Quem?", referindo aqui apenas ao docente e na dimensão "Para que?" referindo-se aos objetivos que este ator pode querer almejar, ambos delineados nos modelos de (Chatti et al., 2012; Gasevic et al., 2017) e ciclos de vida (Clow, 2012; Khalil \& Ebner, 2015) são consolidadas as seguintes ações:

- Adaptação (organizar/moldar de forma adaptativa os recursos de aprendizagem e as atividades);

- Avaliação/Feedback (fornecer informações interessantes geradas com base em dados sobre os interesses do discente e o contexto de aprendizado);

- Benchmarking (identificar as melhores práticas que produzem resultados superiores);

- Intervenção(impedir a evasão, determinar quais discentes podem estar em risco, aconselhar os discentes que podem precisar de assistência adicional e melhorar o sucesso dos discentes);

- Monitoramento (acompanhar as atividades dos alunos e gerar relatórios);

- Personalização (apoiar a aprendizagem para todos os alunos, de forma individualizada);

- Predição (explorar um valor desconhecido, como: desempenho, conhecimento, pontuação ou nota)

- Recomendação (fazer recomendações de novas atividades);

- Reflexão/Iteração (autoavaliar o trabalho passado para melhorar a experiência futura);

- Tutoria (apoiar os discentes na sua orientação e introdução em novos módulos de aprendizagem).

Portanto, fica aqui a consolidação de dez ações provenientes de ferramentas de LA que poderão ser evidenciadas pelo docente em sua prática avaliativa. 


\section{Experimento com ferramentas de Learning Analytics para docentes}

A partir da consolidação das possíveis dez ações docentes perante as ferramentas de LA, foi realizado o experimento com nove docentes de IES em EaD que utilizaram estas ferramentas em suas disciplinas no AVA Moodle, que em seguida foram questionados, sobre qual(is) é(são) o(s) objetivo(s) (ações) evidenciados por cada ferramenta em prol da prática avaliativa docente.

Os dados coletados deste experimento ocorreu durante um curso de capacitação para docentes de IES que atuam na EaD no AVA Moodle realizado entre o período de outubro a março de 2019.

Os nove docentes envolvidos tiveram o seguinte perfil por sexo feminino (60\%) e masculino (40\%); por idade de 31 a 40 anos (60\%), 41 a 50 anos (10\%) e 51 a 60 anos (30\%); e por nível de formação especialista $(20 \%)$, mestre $(60 \%)$ e doutor (20\%). Ambos têm experiência com cursos superiores (sendo licenciatura, bacharelado e/ou tecnólogo) a distância e o tempo médio de atuação em EAD nas IES foi de 5,7 anos entre todos estes envolvidos. Também, entre estes, havia os que já utilizaram os seguintes AVA: Moodle, Teleduc, Aulanet e E-Proinfo.

O AVA Moodle contempla recursos em LA, que já vem sendo trabalhado em vários aspectos desde as versões mais antigas. Além de incorporar ferramentas nativas em seu ambiente, permite também o adicionamento de ferramentas não nativas ( p/ugins) e/ou integração de outros sistemas externos.

Optou-se por ferramentas presentes no site oficial do AVA Moodle (MOODLE, 2017), pois se atribui credibilidade/confiabilidade perante seus desenvolvedores, considerando-se também a possível adesão destas ferramentas por parte de qualquer IES em seus respectivos AVA.

Diante da descrição de ferramentas para LA, foram identificadas as ferramentas que atendeu, inicialmente, ao único critério: "podem ser utilizadas pelo perfil docente", sendo apresentado o resultado na Tabela 1: 
Tabela 1. Ferramentas com recursos de LA para o perfil de docente no AVA Moodle

\begin{tabular}{|c|c|c|c|}
\hline Ferramenta & $\begin{array}{l}\text { Formato de } \\
\text { apresentação }\end{array}$ & $\begin{array}{c}\text { Tipo* } \\
\text { Nativa/Não Nativa }\end{array}$ & Descrição geral \\
\hline Feedback & Atividade & Nativa & $\begin{array}{l}\text { Ferramenta de pesquisa configu- } \\
\text { rável para coleta de feedback }\end{array}$ \\
\hline Survey & Atividade & Nativa & $\begin{array}{l}\text { Conjunto de pesquisas educacio- } \\
\text { nais padronizadas }\end{array}$ \\
\hline Inspire & $\begin{array}{l}\text { Ferramenta } \\
\text { Administrativa }\end{array}$ & Nativa & $\begin{array}{l}\text { LA descritiva e preditiva nativa do } \\
\text { Moodle, a partir da versão } 3.3\end{array}$ \\
\hline Logs & Relatório & Nativa & Log filtrável de eventos \\
\hline Activity & Relatório & Nativa & $\begin{array}{l}\text { Ver contagem de atividades em } \\
\text { curso }\end{array}$ \\
\hline $\begin{array}{l}\text { Activity comple- } \\
\text { tion }\end{array}$ & Relatório & Nativa & $\begin{array}{l}\text { Matriz de conclusão de estudan- } \\
\text { tes e atividades }\end{array}$ \\
\hline Live logs & Relatório & Nativa & $\begin{array}{l}\text { Atualizando automaticamente log } \\
\text { de eventos }\end{array}$ \\
\hline (Quiz) Statistics & Relatório & Nativa & $\begin{array}{l}\text { Relatório de desempenho do quiz } \\
\text { do discente }\end{array}$ \\
\hline $\begin{array}{l}\text { (Course) Partici- } \\
\text { pation }\end{array}$ & Relatório & Nativa & $\begin{array}{l}\text { Participação de discente único } \\
\text { em curso }\end{array}$ \\
\hline Events list & Relatório & Nativa & $\begin{array}{l}\text { Eventos que podem ser monitora- } \\
\text { dos / pesquisados em logs }\end{array}$ \\
\hline $\begin{array}{l}\text { (Gradebook) Over- } \\
\text { view }\end{array}$ & Relatório & Nativa & $\begin{array}{l}\text { Visualização das notas dos dis- } \\
\text { centes em todas as atividades }\end{array}$ \\
\hline Event monitor & Relatório & Nativa & $\begin{array}{l}\text { Ferramenta de monitoração proa- } \\
\text { tiva e focada }\end{array}$ \\
\hline Questionnaire & Atividade & Não Nativa & $\begin{array}{l}\text { Ferramenta de pesquisa configu- } \\
\text { rável }\end{array}$ \\
\hline $\begin{array}{l}\text { Engagement } \\
\text { Analytics }\end{array}$ & $\begin{array}{l}\text { Atividade, blo- } \\
\text { co e relatório }\end{array}$ & Não Nativa & $\begin{array}{l}\text { Relatório de medição de engaja- } \\
\text { mento configurável }\end{array}$ \\
\hline $\begin{array}{l}\text { Progress Bar } \\
\text { (Completion Pro- } \\
\text { gress) }\end{array}$ & Bloco & Não Nativa & $\begin{array}{l}\text { Ferramenta de gerenciamento de } \\
\text { tempo para discentes com visão } \\
\text { geral para professores }\end{array}$ \\
\hline $\begin{array}{l}\text { Configurable Re- } \\
\text { ports }\end{array}$ & Bloco & Não Nativa & $\begin{array}{l}\text { Geração e visualização de rela- } \\
\text { tórios }\end{array}$ \\
\hline Course Dedication & Bloco & Não Nativa & $\begin{array}{l}\text { Tempo estimado online para es- } \\
\text { tudantes }\end{array}$ \\
\hline Graph Stats & Bloco & Não Nativa & Visitas diárias ao site ou curso \\
\hline GISMO & Bloco & Não Nativa & $\begin{array}{l}\text { Numerosos gráficos de partici- } \\
\text { pação na atividade estudantil }\end{array}$ \\
\hline Level Up! & Bloco & Não Nativa & $\begin{array}{l}\text { Medidor de participação de acio- } \\
\text { namento de incentivo }\end{array}$ \\
\hline Analytics Graphs & Bloco & Não Nativa & $\begin{array}{l}\text { Visualização da participação dos } \\
\text { discentes }\end{array}$ \\
\hline Heatmap & Bloco & Não Nativa & $\begin{array}{l}\text { Indicação codificada por cores } \\
\text { das visualizações de atividades na } \\
\text { página do curso }\end{array}$ \\
\hline $\begin{array}{l}\text { Ad-hoc database } \\
\text { queries }\end{array}$ & Relatório & Não Nativa & $\begin{array}{l}\text { Geração e visualização de relató- } \\
\text { rios baseados em SQL }\end{array}$ \\
\hline Forum Graph & Relatório & Não Nativa & Gráfico de interações do fórum \\
\hline Grade distribution & Relatório & Não Nativa & $\begin{array}{l}\text { Visualiza as notas dos alunos em } \\
\text { um curso }\end{array}$ \\
\hline
\end{tabular}

* Nativa: que já vem instalada por padrão no AVA Moodle.

Fonte: Adaptado de Moodle (2017). 
Observa-se portanto, que das 31 ferramentas existentes, 25 ferramentas (tabela 1) dispõem do perfil para servir de apoio ao docente. Destas, 12 são "nativas" e 13 são "não nativas".

Importante destacar também, que no site oficial existem outras ferramentas com recurso de LA, porém exigindo-se integração externa ao Moodle, como pode-se citar o Moodle Activity Viewer ${ }^{5}$, Intelliboard ${ }^{6}$, Analytika ${ }^{7}$, Learning Locker ${ }^{8}$, SmartKlass ${ }^{9}$ e o Moodle Analytics Dashboard (Einhardt et al., 2016) que não se encontra ainda no site, contendo algumas destas versões pagas. Basicamente, são extensões que precisam serem instaladas nos navegadores, a exemplo do Chrome $^{10}$, das máquinas locais.

Para empregar o uso de algumas destas ferramentas, a fim de focar a prática avaliativa diagnóstica docente, foram selecionadas algumas destas ferramentas contidas na tabela 1 . Como critérios iniciais para exclusão desta listagem, temos:

- não exigir a integração externa ao AVA, por entender que estas iriam de alguma forma sofrer alguma resistência pelos docentes;

- ser "não nativa", pois trata-se de ferramentas não existentes por padrão, que potencialmente os docentes ainda não tinham utilizado;

- que tenha relação direta com os objetivos de LA;

- que não tenha similaridade com outras do tipo nativa.

Como resultado destes critérios de exclusão, foram descartadas todas as ferramentas que exigem a integração externa ao AVA, as ferramentas "nativas" segundo a tabela 1 e as ferramentas Questionnaire, pois entendeuse que não havia relação direta com os objetivos de LA e Ad-hoc database queries pela sua similaridade com outras nativas.

Seguindo estes critérios preliminares, chegaram-se então as 13 ferramentas, visualizadas na tabela 2 :

${ }^{5}$ https://damos.world/2013/08/30/the-moodle-activity-viewer-mav-heatmapsof-student-activity/

${ }^{6}$ https://www.intelliboard.net/

7 https://www.zoola.io/

8 https://www.ht2labs.com/learning-locker-community/overview/

${ }^{9}$ https://moodle.org/plugins/local smart klass

${ }^{10} \mathrm{https}: / /$ developer.chrome.com/extensions 
Tabela 2. Ferramentas com recursos de LA para o perfil de docente selecionadas

\begin{tabular}{|c|c|}
\hline Ferramenta & $\begin{array}{c}\text { Número de sites utilizando } \\
\text { (em agosto/2018) }\end{array}$ \\
\hline Configurable Reports & $6439^{i}$ \\
\hline $\begin{array}{l}\text { Progress Bar } \\
\text { (Completion Progress) }\end{array}$ & $3626^{i i}$ \\
\hline Level Up! & $3142^{i i i}$ \\
\hline Course Dedication & $1629^{i v}$ \\
\hline Graph Stats & $885^{v}$ \\
\hline Analytics Graphs & $880^{\text {vi }}$ \\
\hline Heatmap & $343^{\text {vii }}$ \\
\hline GISMO & $342^{\text {viii }}$ \\
\hline Grade Distribution & $250^{\mathrm{ix}}$ \\
\hline Forum Graph & $173^{x}$ \\
\hline Engagement Analytics & $166^{x i}$ \\
\hline 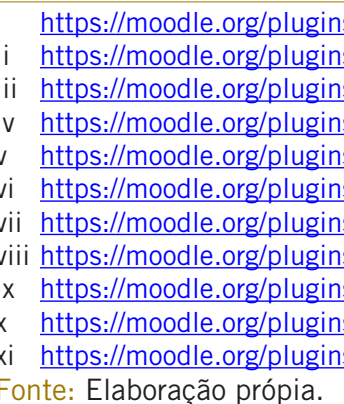 & $\begin{array}{l}\text { sin=block configurable reports } \\
\text { sin=block_completion_progress } \\
\text { sin=block_xp } \\
\text { sin=block dedication } \\
\text { sin=block_graph_stats } \\
\text { sin=block_analytics_graphs } \\
\text { sin=block heatmap } \\
\text { sin=block_gismo } \\
\text { sin=gradereport_gradedist } \\
\text { sin=report forumgraph } \\
\text { sin=block_engagement }\end{array}$ \\
\hline
\end{tabular}

Como critérios finais de exclusão teve a quantidade de sites com o AVA Moodle que utiliza a ferramenta (tabela 2), descartando as 4 ferramentas GISMO, Grade Distribution, Forum Graph e Engagement Analytics e por fim, a exclusão da ferramenta Configurable Reports, que embora tenha alcançado o topo de número de sites que a utiliza, demanda um certo manejo técnico por parte dos docentes para a construção de relatórios personalizados (mesmo sendo destacado que não é exigido conhecimento sobre SQL), sendo entendido que desta forma seria mais direcionado aos gestores.

Portanto, as ferramentas selecionadas foram Analytics Graphs, Course Dedication, Graph Stats, HeatMap, Level Up! e Completion Progress, conforme observado na figura 4. 

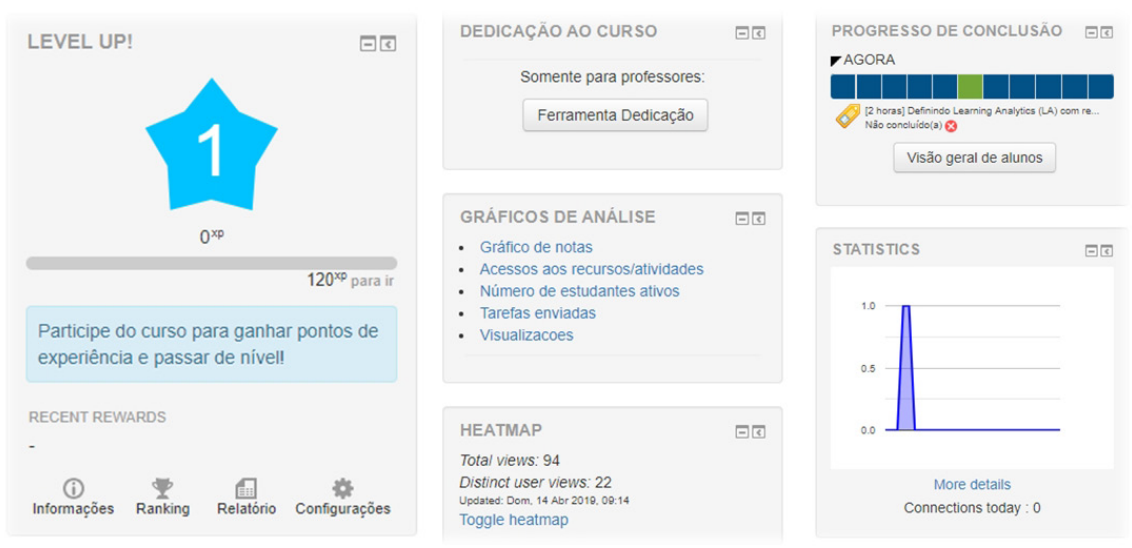

Figura 4. Ferramentas (plugins) utilizados pelos docentes. Fonte: Elaboração própia.

Após selecionar as ferramentas (plugins) (figura 4) atendendo aos critérios estabelecidos, estas foram instaladas em um servidor habilitado contendo o AVA Moodle na versão 3.0.4 ${ }^{11}$ (figura 5), onde estavam inseridas as disciplinas ministradas ${ }^{12}$ de cada um dos docentes envolvidos.

Inicialmente, ainda sem as ferramentas de LA habilitadas, os docentes responderam um questionário semi-estruturado denominado préexperimento ${ }^{13}$. $\mathrm{O}$ objetivo deste questionário foi verificar o seu conhecimento prévio perante o conceito abordado de LA.

A partir das respostas obtidas deste questionário, observa-se no gráfico 1 que 70\% dos docentes informaram que conseguiram, diante de uma definição básica colocada no questionário, identificar com clareza os recursos de LA presentes no AVA Moodle.

11 Optou-se por esta versão, por conta da compatibilidade com as versões dos AVA-Moodle das IES que os docentes envolvidos atuam.

${ }^{12}$ Foi solicitado o arquivo da última disciplina que o docente atuou na IES, assegurando todos os critérios de privacidade e éticos, conforme comitê de ética, com consentimento de ambas as partes envolvidas.

${ }^{13} \mathrm{https}$ ://forms.gle/xT4zgRtUspaP9kme8 


\section{LabExperimento}

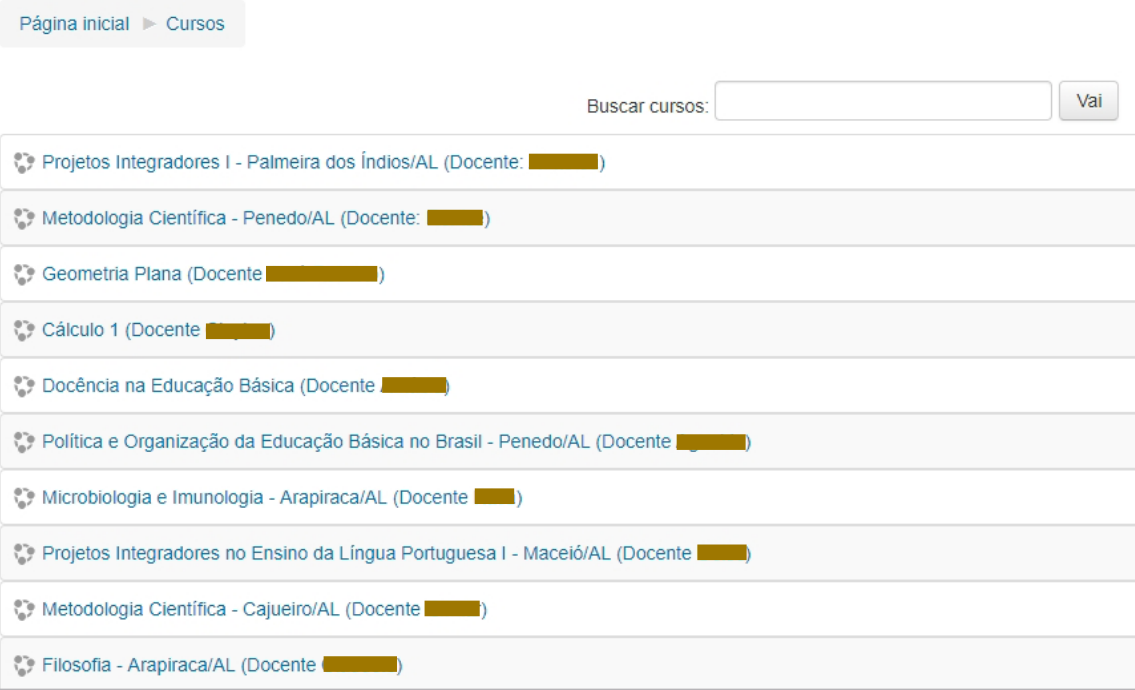

Figura 5. Disciplinas no servidor do AVA-Moodle dos docentes envolvidos. Fonte: Elaboração própia.

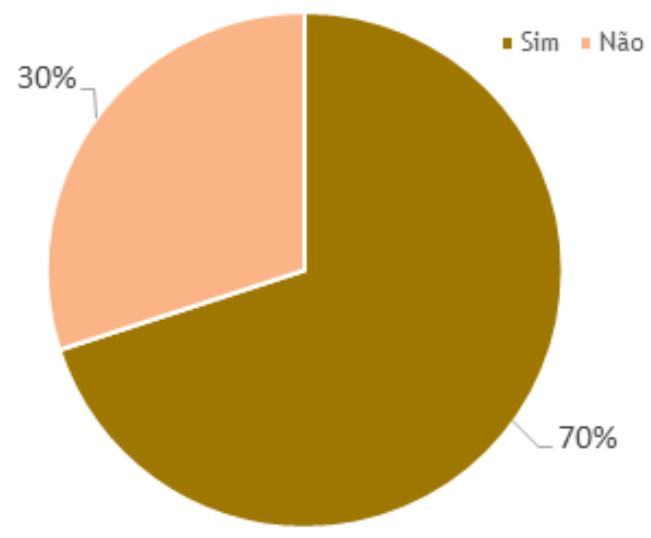

Gráfico 1. Identificação de recursos de LA no AVA-Moodle pelos docentes. Fonte: Elaboração própia. 
Daqueles que informaram que conseguiram identificar os recursos de LA, foram instigados a citar exemplos destes recursos no AVA Moodle que eles atuam. As respostas foram categorizadas sendo evidenciadas na figura 6 .

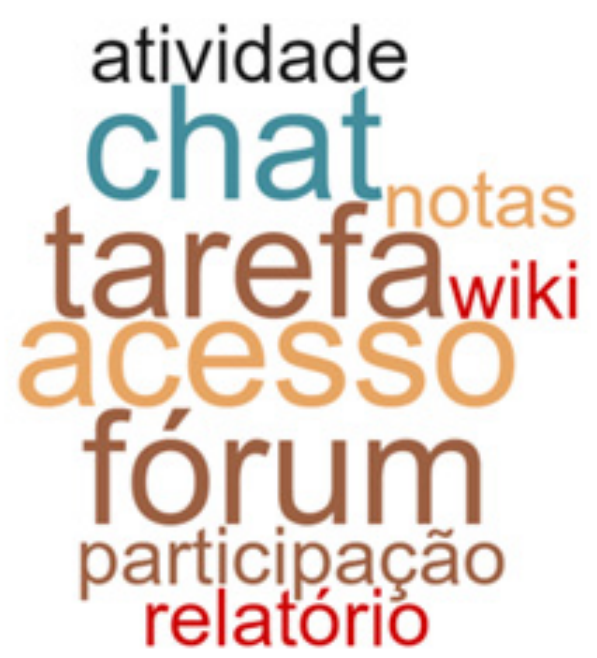

Figura 6. Nuvem de palavras evidenciando os recursos de LA identificados pelos docentes nos AVA que atuam.

Fonte: Elaboração própia.

Observa-se na figura 6 que os recursos identificados foram divergentes quanto ao foco principal da definição básica apresentada: "aprender sobre 0 andamento da aprendizagem de seus discentes", onde se destacam as métricas, no entanto apareceram as interfaces ("fórum", "tarefa", "chat", "wiki" e "atividade") e o "acesso", excetuando-se de forma coerente as palavras "relatório", "notas" e "participação".

Após este diagnóstico inicial, os docentes foram submetidos a uma capacitação/treinamento, sendo mostrado o potencial dos seis plugins selecionados com recursos de LA e habilitaram o uso desses recursos em suas respectivas disciplinas.

Posteriormente, foram motivados a utilizar todos os conhecimentos abordados sobre esses recursos de LA em suas respectivas disciplinas. Após o experimento os docentes responderam outro questionário semi-estruturado denominado pós-experimento ${ }^{14}$.

\footnotetext{
${ }^{14}$ https://forms.gle/h3uGZ8HxUVJFrPWVA
} 
Neste questionário os docentes foram perguntados inicialmente, se já haviam utilizado antes algumas destas ferramentas (Course Dedication, Analytics Graphs, HeatMap, LevelUp!, Completion Progress e Graph Stats), todos que responderam informaram que não. Todos também responderam que acreditam que perante as ferramentas de LA utilizadas é possível se fazer a avaliação diagnóstica.

\section{Resultados do experimento e discussões}

Com base nas possíveis dez ações aqui já expostas, originadas a partir da LA, os docentes informaram diante das seis ferramentas utilizadas, qual ou quais ações, a partir dos objetivos de LA cada uma proporciona, conforme apresentado no gráfico 2 .

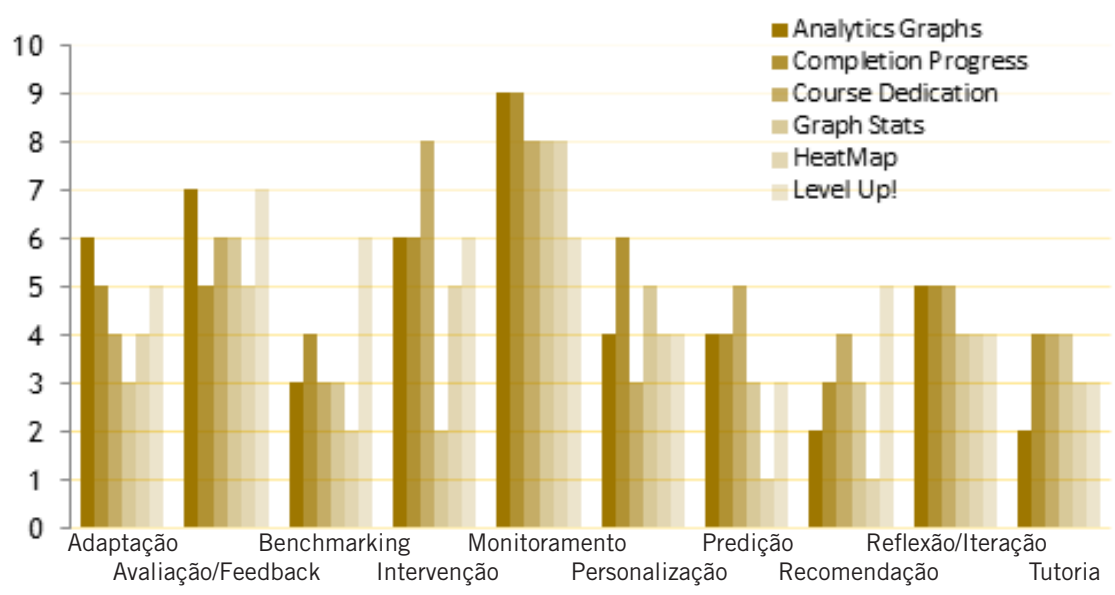

Gráfico 2. Ações a partir dos objetivos de LA proporcionados pelas seis ferramentas utilizadas pelos docentes envolvidos.

Fonte: Elaboração própia.

Nota-se no gráfico 2, que todos os nove docentes envolvidos, sem exceção, identificaram as ferramentas "Analytics Graphs" e "Completion Progress" como ação docente para "monitoramento". 


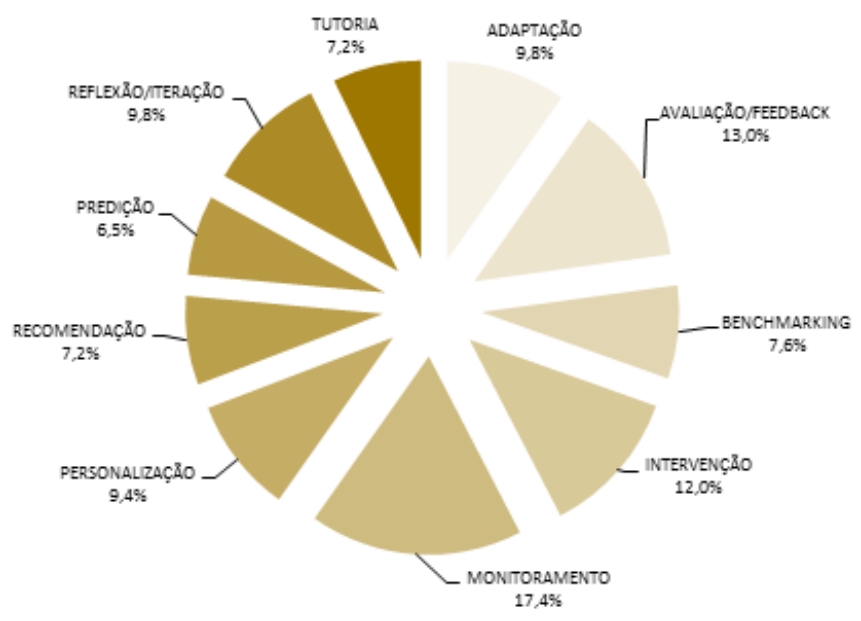

Gráfico 3. Porcentagem de ações da LA perante as ferramentas.

Fonte: Elaboração própia.

No gráfico 3, de forma mais sintetizada no tocante as ações, segundo os docentes envolvidos, diante da sua utilização perante as ferramentas disponibilizadas de LA, oferecem ações com maior grau de "monitoramento" $(17,4 \%)$, seguido por "avaliação/feedback" (13,0\%) e "intervenção" (12,0\%).

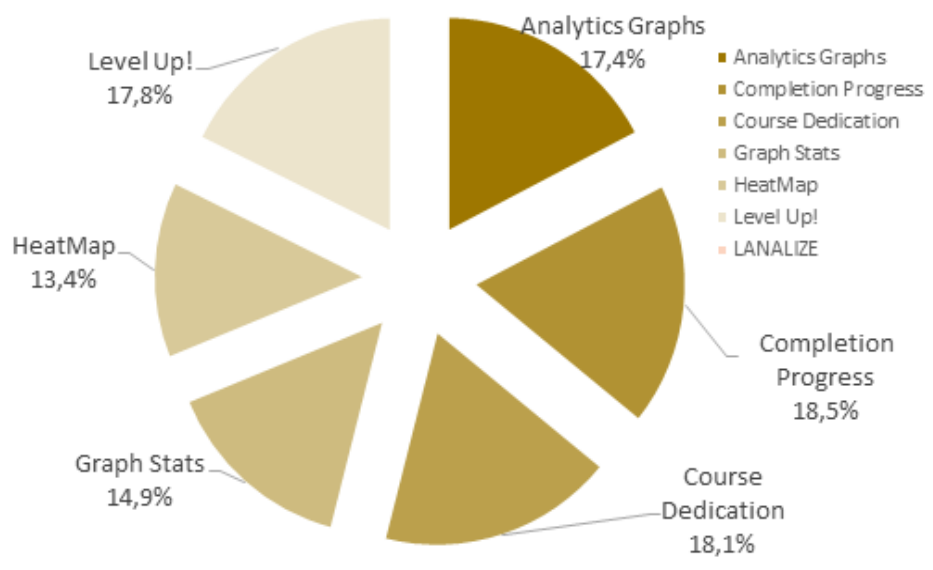

Gráfico 4. Porcentagem correspondente das ferramentas perante as ações de LA. Fonte: Elaboração própia. 
Já no gráfico 4, no qual o foco são as ferramentas que oferecera mais ações, as ferramentas que tiveram maiores destaques foram Completion Progress (18,5\%), Course Dedication (18,1\%) e Level Up! (17,8\%) com mais ações de LA disponibilizadas, segundo os docentes envolvidos.

Embora Chatti et al. (2012) advertem que esses objetivos de LA não são facilmente mensurados, necessitando de mais componentes de indicadores e métricas de desempenho, sendo sugerido, por estes autores, uma definição tripla de objetivo/indicador/métrica, constata-se aqui que segundo a interpretação holística por parte dos docentes envolvidos as ferramentas disponibilizadas e utilizadas dão subsídios para as suas prática avaliativas diagnósticas.

Essa constatação é corroborada pelo resultado obtido pela seguinte pergunta aos docentes envolvidos: "diante de tudo que você já visualizou no curso de capacitação, você acredita que LA pode potencializar a sua prática avaliativa no AVA?", tendo como resultado $88,9 \%$ como sim.

Portanto, de acordo com a consolidação dos objetivos de LA baseandose nos modelos (Chatti et al., 2012) (Gasevic et al., 2017) e ciclos de vida (Clow, 2012; Khalil \& Ebner, 2015) apresentados, a LA representa, diante das evidências identificadas e apresentadas pelos docentes envolvidos, um relevante potencial para otimizar as ações pretendidas a fim de melhorar a eficiência perante 0 andamento de um determinado processo de ensinoaprendizagem.

\section{Considerações finais}

As IES precisam lidar com as tecnologias que incorporam rápidas mudanças, atingindo principalmente os docentes, que segundo Martínez et al. (2018) necessitam incorporar em sua prática, inovações nas metodologias educacionais existentes, em prol da qualidade educacional.

Porém, o uso de uma nova tecnologia não se tem garantia de uma inovação educacional, fazendo-se necessária à vinculação das tecnologias para além de metodologias, com práticas e processos de mediação pedagógica (Saccol et al., 2011). Conforme afirmam Vosgerau et al. (2016, p. 114) "adotar, adaptar e apropriar as tecnologias na educação, em qualquer modalidade, é uma urgência, que deverá ser planejada por equipes interdisciplinares". 
Silva (2015) destaca que é vantajoso o domínio dos docentes na interação com as tecnologias atuais, periciando a prática na educação online a partir dos AVA, embora a falta de capacitação mais robusta, incluindo a acompanhamento científico de todos os temas relacionados a pedagogia em prol da educação, elimina o contexto geral proposto por um planejamento técnico-pedagógico.

O AVA Moodle possibilita a integração de algumas ferramentas, como plugins e sistemas externos, capazes de promover a integração entre a LA, que tem sido categorizadas com diferentes níveis de aprofundamento em suas referidas integrações com os AVA.

Diante da tecnologia, tendo incorporado recursos de LA, o AVA representa um aliado para o apoio as ações docente. Porém, é preciso analisar a partir das duras críticas que a área da tecnologia na educação enfrenta quanto à carência de fundamentação teórica.

A LA nas IES proporciona além dos benefícios de refletir a aprendizagem do discente, fornecendo importantes subsídios para os docentes, também pode trazer cooperação interinstitucional (Leitner et al., 2017). Fica evidenciado que a LA é vista como um grande aliado para a melhoria da qualidade da educação. Porém, conforme é percebido por Trigt (2016) é que o tempo dirá se a customização da educação em LA a partir dos dados dará certo ou não, embora se tenha razões suficientes para acreditar neste incremento para a educação, em especial a de nível superior.

\section{Referências bibliográficas}

ABED (2017). Censo EAD.BR: relatório analítico da aprendizagem a distância no Brasil 2016. ABED. Curitiba: InterSaberes.

Bitencourt, B. M., Severo, M. B., \& Gallon, S. (2013). Avaliação da aprendizagem no ensino superior: desafios e potencialidades na educação a distância. Revista Eletrônica de Educação, 7(2), 211-226.

Chatti, M. A., Dyckhoff, A. L., Schroeder, U., \& Thüs, H. (2012). A reference model for learning analytics. International Journal of technology enhanced learning (IJTEL) - Special Issue on "State-of-the-art in TEL", 1-22.

Clow, D. (2012). The learning analytics cycle: closing the loop effectively. Proceedings...LAK '12 Proceedings of the 2nd International Conference on Learning Analytics and Knowledge, 134-138.

Daniel, B. (2015). Big Data and analytics in higher education: Opportunities and challenges. British Journal of Educational Technology, 46(5), 904-920. 
Dodun, O., Panaite, E., Seghedin, N., Nagît, G., Dusa, P., Nestian, G. Slatineanu (2015). Analysis of an e-learning platform use by means of the axiomatic design. In: 9 . International conference on axiomatic design (ICAD 2015). Proceedings...Florence, Italy, v. 34, p. 244-249.

Einhardt, L., Tavares, T.A., \& Cechinel, C. (2016, October). Moodle analytics dashboard: a learning analytics tool to visualize users interactions in moodle. In XI Latin American Conference on Learning Objects and Technology (LACLO) (pp. 1-6). IEEE.

Ekuase-Anwansedo, A., Craig, S. F., \& Noguera, J. (2018). How to survive a learning management system (LMS) implementation? A stakeholder analysis approach. Proceedings...18th SIGUCCS, p. 165-168.

Gasevic, D., Kovanovic, V., \& Joksimovic, S. (2017). Piecing the learning analytics puzzle: a consolidated model of a field of research and practice. Journal Learning: Research and Practice, 3, 63-78.

Khalil, M., \& Ebner, M. (2015). Learning analytics: principles and constraints. Proceedings of world conference on educational multimedia, hypermedia and telecommunications, 1326-1336.

Leitner, P., Khalil, M., \& Ebner, M. (2017). Learning analytics in higher education: a literature review. In: A. Peña-Ayala (ed.) Learning analytics: fundaments, applications, and trends. Springer international publishing, 1-23.

Machado, L. R., Longhi, M. T., \& Behar, P. A. (2013). Domínio tecnológico: saberes e fazeres na educação a distância. In: P. A. Behar, Competências em educação a distância (pp. 56-80). Porto Alegre: Penso.

Iglesias, M.J., Lozano, I., \& Roldán, I. (2018). La calidad e innovación educativa en la formación continua docente: un estudio cualitativo en dos centros educativos. Revista Iberoamericana de Educación, 77(1), 13-34. Disponível em: https://bit.ly/2UXeBRd

Medio, C. de, Gasparetti, F., Limongelli, C., Sciarrone, F., \& Temperini, M. (2017). Course-Driven Teacher Modeling for Learning Objects Recommendation in the Moodle LMS. In: UMAP'17 Adjunct, Bratislava, Slovakia. Proceedings...Bratislava, p. 141-145.

MOODLE. Learning Analytics. 2017. Disponível em: https://bit.ly/2KxaqHX.

Peña-Ayala, A. (2017). Learning analytics: fundaments, applications, and trends: a view of the current state of the art to enhance e-learning. Cham: Springer.

Polak, Y.N. (2009). A avaliação do aprendiz em EAD. In: F. M. Litto, \& M. Formiga, (orgs.). Educação a distância: o estado da arte (pp. 153-160). São Paulo: Pearson Education do Brasil, .

Saccol, A., Schlemmer, E., \& Barbosa, J. (2011). M-learning e u-learning: novas perspectivas das aprendizagens móvel e ubíqua. São Paulo: Pearson Prentice Hall.

Siemens, G. (2010). What are learning analytics? In: Elearnspace: learning, networks, knowledge, technology, community. Disponível em: https://bit.ly/2PcZKQE.

Siemens, G. (2013). Learning analytics: the emergence of a discipline. Journal American Behavioral Scientist, 57(10), 1380-1400.

Silva, A.C., Leite, L.S., \& Silva, C.M. (2009). Avaliação da aprendizagem em ambientes virtuais: é possível inovar? Revista Meta: Avaliação, Rio de Janeiro, 1(2), 237-248.

Silva, R.S. (2013). Gestão de EaD: educação a distância na era digital. São Paulo: Novatec. 
Silva, R. S. (2015). Ambientes virtuais e multiplataformas online na ead. São Paulo: Novatec.

Trigt, M. V. (2016). How data can improve the quality of higher education. SURFnet.. Disponivel em: https://bit.ly/2GhZ2vn.

Vosgerau, D., Brito, G. S., \& Camas, N. (2016). PNE 2014-2024: Tecnologias educacionais e formação de professores. Revista Formação Docente, 8(14),103-118. 